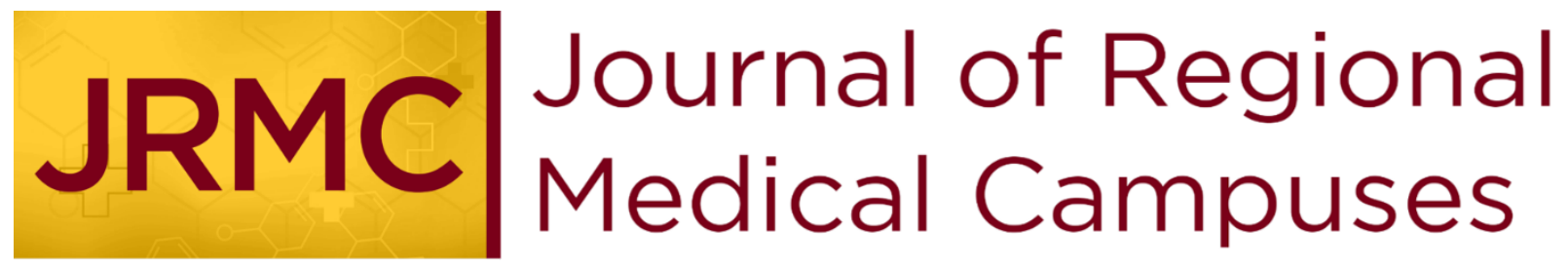

Inoculating Trainees Upstream: What Distressed Providers Wished They'd Learned in Medical School

Linda L.M. Worley, MD, FACLP

z.umn.edu/JRMC

All work in JRMC is licensed under CC BY-NC

(c) $\underset{\mathrm{BY}}{\mathrm{NC}}$ 


\section{Inoculating Trainees Upstream: What Distressed Providers Wished They'd Learned in Medical \\ School}

Linda L.M. Worley, MD, FACLP

\section{Topic:}

Deciphering the secrets for preventing burnout, distressed, disruptive physician behavior and the tragic of loss of life to suicide couldn't be more important for the overall health of our nation than now as we face an increasingly critical physician shortage.

Physicians mandated to attend the Vanderbilt Center for Professional Health, Distressed Physicians' Course often as a result of their disruptive behavior offer invaluable lessons underlying the core drivers beneath their distress. Armed with these insights, it is possible to design and implement upstream educational curricula for medical students, residents, and early career faculty to inoculate them proactively to better navigate the challenges in medicine today.

\section{Short Description:}

The stressors in medicine today are palpable. Record numbers of physicians report significant symptoms of burnout with approximately 400 physicians per year choosing to end their lives by suicide. Inner distress often manifests outwardly through disruptive behavior potentially jeopardizing the overall safety and functioning of the healthcare team.

The Vanderbilt Center for Professional Health Distressed Physicians' Course remediates physicians who are referred as a result of their disruptive, distressed behavior.

The physicians referred to the course are highly skilled, productive physicians who graduated near the top of their class matching into highly competitive specialties. They offer invaluable insights into the shortcoming of their medical training. Course faculty member and Associate Regional Dean of UAMS Northwest College of Medicine, Linda L.M. Worley, MD, is infusing this missing curriculum into the training of regional medical students, residents and staff.

\section{Four questions that were posed to/considered by session participants:}

1) How many physicians will we be short when looking ahead to the projected needs by 2030?

2) What are the stressors driving physician burnout, early retirement, and suicide?

3) What are distressed-disruptive physician behaviors and how do they negatively impact the performance of the healthcare team?

4) What are the essential skills physicians in training need to master in order to avoid becoming a distressed, disruptive provider?

\section{Three take home points from session:}

1) Physicians mandated to attend a course for distressed-disruptive behavior reported minimal prior training in team behavior, managing stress and burnout, conflict management, mindfulness, and emotional intelligence.

2) Disruptive physician behavior is unacceptable; it decreases patient satisfaction, increases the frequency of medical errors and litigation, creates a toxic workplace with costly turnover, and contributes to low morale and difficulty recruiting.

3) Physicians have exceedingly high standards and struggle when others fail to live up to their expectations. They are highly skilled, productive, driven, and dedicated to perfection in patient care. Building leadership skills of self-awareness, selfcontrol, and empathy, including an ability to effectively listen and resolve conflict while still in training, will prepare them to more effectively navigate the stressful healthcare environment without becoming a distressed-disruptive provider

Linda L.M. Worley, MD, FACLP; Associate Regional Dean, University of Arkansas for Medical Sciences Northwest Campus College of Medicine, Professor of Psychiatry and Obstetrics and Gynecology, UAMS, Adjunct Professor of Medicine, Vanderbilt Center for Professional Health, WorleyLindaL@uams.edu 